EOMmun Communication et organisation

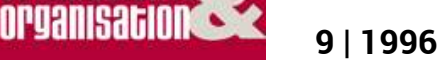

La communication des institutions religieuses

\title{
La communication des télévangélistes
}

\section{Hugues Hotier}

\section{OpenEdition}

Journals

Édition électronique

URL : http://journals.openedition.org/communicationorganisation/1843

DOI : 10.4000/communicationorganisation. 1843

ISSN : 1775-3546

\section{Éditeur}

Presses universitaires de Bordeaux

\section{Édition imprimée}

Date de publication : 1 mai 1996

ISSN : 1168-5549

\section{Référence électronique}

Hugues Hotier, "La communication des télévangélistes », Communication et organisation [En ligne], 9 | 1996, mis en ligne le 26 mars 2012, consulté le 30 avril 2019. URL : http://journals.openedition.org/ communicationorganisation/1843; DOI : 10.4000/communicationorganisation.1843

Ce document a été généré automatiquement le 30 avril 2019

(c) Presses universitaires de Bordeaux 


\title{
La communication des télévangélistes
}

\author{
Hugues Hotier
}

1 Le fait de n'être ni favorable ni hostile aux institutions religieuses pourrait garantir au chercheur une accession relativement aisée à l'indispensable neutralité qui sied aux sciences humaines. Mais l'observation des entreprises - car il s'agit bien d'entreprises religieuses que dirigent les "télévangélistes » est suffisamment déconcertante pour troubler l'esprit et rendre la distanciation difficile.

2 Les hommes et les femmes qui exercent leur sacerdoce sur les chaînes de télévision américaines sont dénommés par ce mot nouveau qui désigne à la fois le message et le médium. Succédant aux prédicateurs qui, dès le XviII ${ }^{\mathrm{e}}$ siècle arpentaient les rues des villes et les chemins des campagnes, ils utilisent le moyen le plus moderne et le plus efficace qui soit pour convertir avant de baptiser ou rebaptiser (on dit alors de ces nouveaux élus qu'ils sont «born again ») dans leurs temples. Les télévangélistes sont tous chrétiens et la Bible est leur référence. Ils sont fondamentalistes et, au regard de la politique, appartiennent dans une proportion très majoritaire à la droite la plus radicale. Ainsi militent-ils contre l'interruption volontaire de grossesse, qu'ils appellent holocauste, avec une violence, au moins verbale, inouie. Comme la plupart des intégristes, ils tentent, avec assez bien de succès, de pénétrer le pouvoir politique avant de se l'approprier. Reagan et Bush $^{1}$ avaient des « born again » dans leur proche entourage et Ingrid Carlander ${ }^{2}$ affirme que certaines décisions prises par l'ONU sous l'impulsion des États-Unis et qui n'allaient pas dans un sens progressiste avaient été dictées par ces conseillers born again. Ils sont quarante millions d'Américains ainsi «nés à nouveau » et ils contituent un groupe de pression de plus en plus puissant et d'autant plus imprévisible que ses leaders tiennent leurs ordres directement de Dieu. Ainsi, en 1988, Dieu avait-il intimé au télévangéliste Pat Robertson de se présenter à l'élection présidentielle. Ce fut néanmoins George Bush qui emporta la course à la candidature.

3 Pour comprendre la floraison de ces églises bâties par un homme qui en est à la fois le gourou et le chef d'entreprise - il n'y en a pas moins de quatre cents aux États-Unis - il faut tenir compte de deux éléments que rappelle Ingrid Carlander. L'un est historique, l'autre est politique. 
4 Les Puritains du XVII siècle étaient, à l'instar des Juifs, persuadés que «Dieu avait signé un pacte avec l'Amérique». Ce qui fait que toute réussite terrestre pouvait être interprétée comme une production de la volonté divine. N'acceptant pour autorité que la Bible et communiquant directement avec Dieu, ils s'opposaient de fait à l'Église anglicane, émanation et, dans un certain sens, symbole de la puissance tutélaire et colonisatrice puisqu'ils se dispensaient de toute intercession religieuse. Ce principe est resté à la base des religions baptiste et pentecôtiste ainsi que d'autres petites religions. Mais l'austérité de la foi puritaine avait de quoi décourager plus d'un et la pensée puritaine périclita. Elle laissa la place pour les premiers prédicateurs qui prêchaient le «Grand Réveil » et qui, par leurs talents d'orateurs, parvinrent à réunir des foules considérables pour l'époque. Ainsi, cite-t-on ce George Whitefield qui venait d'Angleterre et qui prêcha chaque jour devant des foules de 8000 personnes durant six semaines. C'est lui qui importa aux ÉtatsUnis cette immense fête, mi-spectacle mi-office religieux, qu'on appelle revival et qui, parfois sous le nom de camp meeting (convention), draine à l'heure actuelle des foules considérables vers les églises des télévangélistes. Ces revivals ont toujours été le théâtre d'extravagances de fidèles qui, sous l'emprise de la musique et du verbe, ne pouvaient plus se dominer et entraient dans de véritables transes. Les pentecôtistes, particulièrement, s'abandonnent à la glossolalie, c'est-à-dire qu'ils parlent spontanément des langues étranges plus qu'étrangères que personne ne comprend. «Parler en langues » est un don de Dieu qui témoigne de sa puissance et rapproche le fidèle des apôtres qui, le jour de la Pentecôte, reçurent de l'Esprit-Saint le don des langues... Quoi qu'il en soit, on voit que la tradition de la prédication et de ses effets est ancienne et qu'elle constitue un fondement pour les pratiques que nous observons actuellement.

Le Premier Amendement de la Constitution des États-Unis définit la séparation de l'Église et de l'État. Il dit que l'État ni ne s'opposera à la création de religions ni n'en favorisera ou n'en créera lui-même. De cet amendement découle un avantage fiscal particulièrement intéressant pour les églises : les revenus du culte ne sont pas imposables. Il s'agit bien des dons et non, comme certains télévangélistes l'affirmèrent longtemps, de tous les revenus y compris les bénéfices tirés des ventes de livres, de cassettes, de carnets de recettes de cuisine et autres crayons, bonbons, etc. à l'effigie du prédicateur! En d'autres termes, l'argent que l'on a obtenu des fidèles pour évangéliser l'ancienne URSS n'est ni imposable ni contrôlable.

6 Et, de fait, vu de l'extérieur, il semble que ces églises aient pour activité principale d'assurer des revenus élevés à leurs gourous et à leurs familles. Il ne faudrait cependant pas négliger l'apport de ces églises à leurs adhérents. Cet apport se fait essentiellement sous la forme d'offices qui sont de véritables spectacles fondés sur le charisme du prédicateur vedette.

7 Pour résumer, on pourrait dire que les deux mots qui rendent le mieux compte de la réalité communicationnelle des télévangélistes sont charisme et marketing. Nous nous proposons de les développer au travers de la description d'un office à la Family Worship Center de Baton Rouge, Louisiane, d'une part et de l'étude de la collecte de fonds telle qu'elle se pratique le plus souvent chez les télévangélistes. 


\section{Un office à la Family Worship Center de Baton Rouge (Louisiane)}

8 La Family Worship Center est l'église de Jimmy Swaggart qui fut connu en France pour avoir manqué un contrat avec une chaîne de télévision à la suite d'un scandale sexuel. L'église se trouve à Baton Rouge. Elle est sise sur un vaste terrain qu'occupent également un "collège " biblique formant des pasteurs, une maison d'éditions, une maison de production de disques et cassettes sonores ainsi qu'une maison de production de cassettes video et des studios de télévision. L'importance de l'ensemble justifie qu'une des sorties de l'autoroute qui vient de La Nouvelle Orleans lui soit dédiée.

Pour une bonne compréhension de la relation que le télévangéliste entretient avec ses ouailles, il nous faut développer deux points de la vie de Jimmy Swaggart qui constituent des éléments du contexte de l'office que nous nous proposons d'analyser ci-après: la mission divine de 1982 et la tentation satanique de 1988.

Tout débuta par un ordre divin. Le 1er juillet 1982 à 9 heures 15 du matin, alors qu'ils cheminait le long d'un champ de coton prêt à être moissonné, Swaggart eut une vision. Dieu lui déclara qu'il avait l'intention de détruire l'Amérique mais qu'il avait décidé de reporter Armagedon pour permettre à celui qu'il avait choisi, lui-même, de prêcher l'Évangile au monde entier par le moyen de la télévision. « Dieu m'a dit que je devais aller à la rencontre du monde. Il m'a donné mission d'aller à la rencontre du monde par la télévision. Bien sûr, nous construirons des églises et des écoles bibliques mais Il m'a dit que la télévision permettrait d'atteindre des millions et des centaines de millions de personnes et de les atteindre rapidement. Voilà ce qu'il m'a ordonné de faire et que j'essaie de mener à bien. J'ai besoin de votre aide. Et Il m'a dit que vous m'aideriez. Vingt dollars permettront d'atteindre 40000 personnes, 50 dollars permettront d'atteindre 100000 personnes, 100 dollars permettront d'atteindre 200000 personnes, 500 dollars permettront d'atteindre un million de personnes, 1000 dollars permettront d'atteindre 2 millions de personnes ${ }^{3}$ ». Le magazine louisianais Gris Grisqui cite ces paroles fondatrices ajoute qu'elles permirent en trois ans d'acquérir pour 8,5 millions de dollars d'équipement de télévision. Après les avoir prononcées, Swaggart s'assit au piano et, avant de chanter Les champs sont blancs, voici venu le temps de la moisson,il ajouta : «Dieu a laissé la porte ouverte et vous devez nous aider à la maintenir ouverte. Jamais dans l'histoire du monde la porte n'a été ainsi ouverte. Faites un chèque pour les âmes perdues ». 


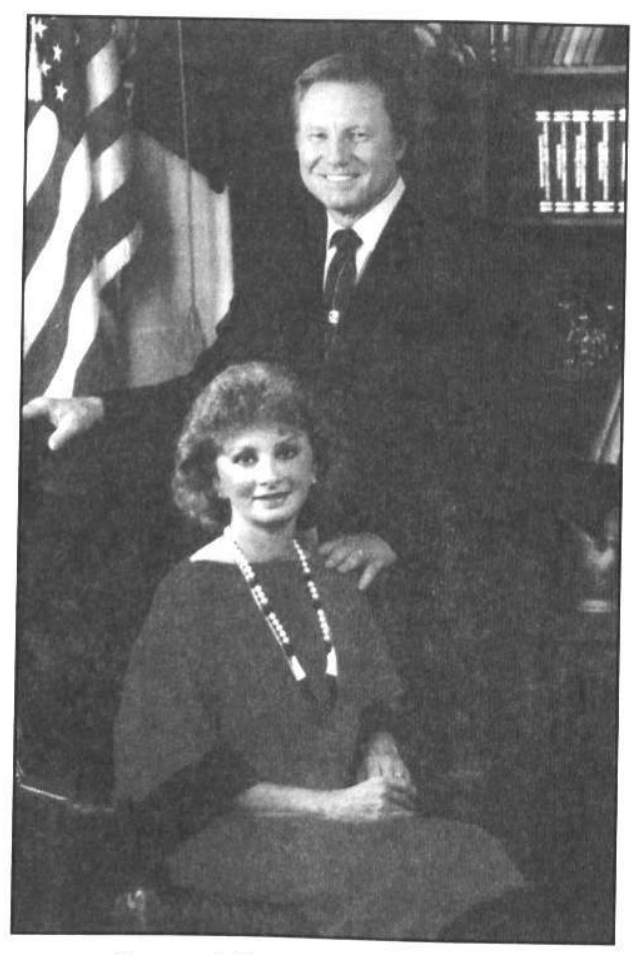

Jimmy et Frances SWAGGART

11 Ainsi débuta la mission de l'un des télévangélistes les plus populaires des États-Unis. En 1985, la Chambre de Commerce de Baton Rouge estimait l'apport économique direct ou induit de cette croisade: création de 751 emplois de pasteurs et de 1502 emplois de techniciens, commerciaux et divers, 33,1 millions de dollars en dépenses directes, 15,75 millions de dollars en salaires et 17,2 millions de dollars en dépôts bancaires. Les impôts collectés par l'État durant la même période se sont élevés à 3,3 millions de dollars ${ }^{4}$

Mais le malin s'en mêla. Et les ennemis que Jimmy Swaggart s'était faits dans sa croisade contre le péché lui prêtèrent main forte. Il avait notamment vilipendé deux télévangélistes aussi célèbres que lui. Il y eut, en 1987, Jim Bakker, dont il dénonça l'adultère avec une prostituée, qu'il accusa d'homosexualité et dont la vie de prédicateur vedette se termina par une condamnation à 45 ans de prison pour fraude fiscale et détournements de fonds. Il y eut, la même année, Marvin Gorman, son concurrent direct, installé à La Nouvelle Orléans, dont il dénonça les turpitudes sexuelles, qu'il accusa d'avoir séduit une de ses paroisiennes et qui finit par être défroqué par les Assemblées de Dieu, sorte de conseil de l'ordre des églises pentecôtistes. Même si le dénonciateur fut condamné à lui payer 10 millions de dollars au titre des dommages et intérêts pour diffamation, Marvin Gorman ne retrouva pas sa situation antérieure. C'est son fils qui fit surprendre et photographier en flagrant délit de relations sexuelles avec une prostituée le pasteur Swaggart. Les médias relatèrent l'affaire et, comme souvent dans ces cas là aux Etats-unis, la prostituée se répandit en interviews. Est-il besoin d'indiquer que cet incident eut des conséquences fâcheuses sur une entreprise entièrement construite autour d'un homme. Le 21 février 1988, Jimmy Swaggart, dans son église de Baton Rouge, devant les caméras de télévision de sa propre production, fait amende honorable. Il reconnaît avoir péché, il demande pardon à sa femme, aux autres pasteurs, aux étudiants de son collège biblique, à son Sauveur et à ses collègues télévangélistes: "Aux Assemblées de Dieu, à cette paroisse, ce collège biblique, ces étudiants, ces professeurs, 
ces choristes et ces musiciens, je demande pardon ${ }^{5}$. " Les Assemblées de Dieu ne trouvèrent pas cette amende honorable suffisante et infligèrent à Swaggart une sanction lourde : deux ans de suspension. Même si swaggart finit par passer outre l'interdiction, se retirant ainsi de la famille pentecôtiste, et reprit sa place à la tête de son église, le préjudice fut considérable ainsi que le rapporte Time: «Le facinant orateur de Baton Rouge était le №1 des prédicateurs mais la chance de Jimmy Swaggart s'est effondrée après qu'il eut été surpris avec une prostituée. Les Assemblées de Dieu l'ont défroqué et son audience télévisuelle a chuté des deux tiers. La construction de son collège biblique est arrêtée et les inscriptions y ont diminué de manière catastrophique. Aujourd'hui, les dons que reçoit Swaggart, 54 ans, n'atteignent probablement qu'un million de dollars par semaine contre trois dans ses jours fastes. Le personnel a été réduit des deux tiers et Swaggart doit faire face à une accumulation de problèmes juridiques ${ }^{6}{ }$.

À noter que, ayant à nouveau été convaincu de relations avec une prostituée - c'était en octobre 1991 en Californie - Swaggart cette fois-ci déclara que Dieu lui avait dit: «Tes affaires ne regardent personne ${ }^{7} »$. Nous devons à la vérité de dire que beaucoup d'employés de la galaxie Swaggart que nous avons rencontrés à Baton Rouge en décembre 1992, nous ont déclaré travailler pour gagner leur vie mais avoir perdu la foi en leur prédicateur.

\section{Office du lundi 3 juillet 1989 (« Monday night service »)}

\section{Le lieu de l'office}

14 Le public est installé sur des sièges disposés en gradins. Les sièges sont répartis en cinq quartiers de trois cent cinquante places chacun, séparés par des allées dont le tracé blanc tranche sur le tapis grenat du sol et de la scène. Un balcon ceinture la salle et peut recevoir plusieurs centaines de personnes. Toutes les trois rangées, le bord du rang est occupé par un membre de l'organisation, en veste rouge. Ces employés veillent à ce que le comportement des assistants reste dans les convenances permises. L'amphithéâtre descend autour d'une piste octogonale surélevée s'avançant en proscénium d'un plateau de forme trapézoïdale dont la petite base jouxte l'un des côtés de la piste octogonale.

Dans le fond de la scène, sur quatre rangs en gradins, une bonne centaine de choristes chantent debout et s'assoient quand le public est invité à le faire, pour écouter le sermon par exemple. La majorité d'entre eux est devant le mur central mais il existe une allée de part et d'autre de ce mur et trois rangs de choristes sont à droite de cette allée ou, si l'on peut recourir au lexique du théâtre, côté cour.

À gauche du chœur, à partir du troisième rang en partant du fond de la scène, est installée une formation orchestrale. Côté jardin, sur des podiums en terrasses, en allant du plus haut au plus bas, se trouvent un orgue Hammond de grande taille, un piano à queue (queue complète), un ensemble de synthétiseurs. À même le plateau, sans podium, les percussions. De l'autre côté de l'allée qui jouxte la partie centrale à gauche de celle-ci, toujours côté jardin, sont quatre trompettes et un saxophone ténor. Au centre, sont assis quatre guitaristes dont l'un, à l'occasion, joue aussi l'harmonica. À l'extrême droite, parmi les invités installés sous les choristes, est un trompettiste. C'est donc une grande formation, forte de quatorze musiciens, qui accompagne l'office.

Devant les guitaristes, donc au milieu du fond formé par le chœur, debout, sont quatre choristes qui, contrairement aux autres, chantent devant des microphones. Les deux 
hommes et les deux femmes qui forment ce petit chœur sont placés sur un seul rang et en alternant les sexes. Ils chantent toujours avec le soliste, même quand le grand chœur est muet.

Rien ici n'évoque un lieu de culte : ni crucifix, ni autel, par exemple. En revanche, sur la partie octogonale de l'ensemble scénique se trouve un pupitre fait d'une plaque oblique, fabriquée dans un matériau transparent, fixée sur un pied métallique.

De telles dispositions scéniques sont connues depuis longtemps puisque les cirques de la fin $d u \mathrm{XVIII}^{\mathrm{e}}$ siècle et de la première moitié du XIX les utilisaient pour donner des spectacles de pantomime dans lesquels hommes et bêtes voisinaient. Les thèmes de ces pantomimes étaient la plupart du temps liés à l'actualité épique (hommage à Napoléon, conquête de l'Algérie, condamnation de la Révolution française dans les cirques britanniques...). L'usage d'une scène en arrière de la piste était rendu nécessaire par cette cohabitation des personnages humains et des acteurs animaux. Ainsi voyait-on, par exemple, une armée de figurants, en retrait sur la scène, encourager un officier aux prises avec un groupe de lions dont les évolutions avaient lieu dans la piste. On peut observer aussi que cet ensemble constitue deux lieux pour deux niveaux du spectacle : un avant plan où se passe l'essentiel de l'action, un arrière plan qui est tantôt un lieu d'accompagnement passif (figuration) ou actif (chœur) et tantôt un lieu de repos, de retraite, pour les protagonistes. Ce qui est notable c'est que, dans cette configuration, les acteurs sont toujours présents à la vue du public. 11 n'y a pas de coulisses où se retirer. Pendant toute la durée du spectacle, les acteurs qui n'agissent pas directement continuent, par leur présence, de participer à l'action dont il sont les témoins. Leurs réactions - étonnement, joie, angoisse, approbation - constituent une partie, muette, du dialogue. Ils contribuent à créer ou à renforcer l'émotion du public et l'on connaît la puissance du non verbal dans ce genre de situation. Nous avons, dans une étude consacrée à la communication au cirque, montré qu'une des spécificités de ce lieu et de ce spectacle était que la disposition en amphithéâtre faisait de chaque spectateur un élément du dit spectacle ${ }^{8}$. Chacun est un figurant pour l'autre et les émotions des uns contribuent à créer celles des autres. Le même constat pourrait s'appliquer ici en raison de la disposition de la salle en amphithéâtre. Mais, en plus, il existe un public d'acteurs montré ostensiblement au public des fidèles en raison de la place qu'on lui assigne en « fond de scène ». C'est, en quelque sorte, un décor vivant qui est ainsi installé à la vue des fidèles.

\section{Les acteurs}

Les choristes, hommes et femmes, sont vêtus de longues robes blanches, amples, à plis verticaux. Le col de ce vêtement est bleu. Il y a donc un effet d'uniformité qui est contredit par le comportement des choristes. Certes, chacun reste à sa place - encore verra-t-on une femme quitter son poste pour courir dans la salle, bras levés, comme prise par la contagion d'une ambiance chaude créé par l'orchestre et les chanteurs - mais les mouvements des corps, du déhanchement à la gestuelle, affichent une grande diversité. Comme si la spontanéité des comportements s'opposait à la statique imposée par l'institution.

21 Les musiciens sont en costume beige pour ceux qui sont à l'extrême gauche, chemise blanche, cravate. En bleu marine pour ceux qui sont dans la partie centrale et pour le trompettiste installé parmi les invités (voir ci-après). Les chanteurs, solistes et membres 
du petit chœur, sont en costume bleu marine et cravate ; les deux femmes du petit chœur sont en robe longue claire.

Jimmy Swaggart est, en 1989, un homme de cinquante-quatre ans. L'impression qui domine quand on le voit est celle d'un individu soigné. Son léger embonpoint est compensé par la qualité du costume gris-bleu bien taillé, les cheveux blonds sont coiffés avec soin et, grâce à un fixateur approprié, leur gonflant du type de celui que les coiffeurs obtiennent par la technique dite du brushing, ne souffre pas lorsqu'il bouge, même si ses mouvements sont brusques et saccadés. La cravate mauve à motifs géométriques tranche sur une chemise blanche. Il porte de fines lunettes à monture métallique dorée, une large alliance d'or ciselé à la main gauche et une gourmette d'or au poignet droit. Nous supposons qu'il s'agit d'or en raison de la couleur et de l'éclat du métal, en raison aussi du fait que ces bijoux sont généralement réalisés dans ce métal. Quoi qu'il en soit, l'effet visuel produit est celui du précieux métal. La cravate est fixée à la chemise par une pince placée au milieu de la poitrine. Il est chaussé de mocassins de couleur marron fins et brillants. Son visage, rond et assez gros avec des yeux un peu enfoncés est assez inexpressif. Il manque de finesse et de nombreux journalistes ont parlé de visage de boxeur.

Sous les gradins des choristes qui sont à l'extrême droite (côté cour, à droite de l'allée), une vingtaine d'invités ont pris place sur des sièges alignés sur quatre rangées. La première de ces rangées est occupée par Jimmy Swaggart et son épouse Frances. Elle restera assise en permanence à cette place, bien en vue.

\section{Le déroulement de l'office}

24 L'office s'articule en trois parties qui ont chacune une dominante de contenu et de forme. La première est un spectacle de gospel où dominent trois chanteurs, accompagnés par un grand orchestre de jazz et des choristes. La seconde consiste en l'interprétation d'une chanson par Jimmy Swaggart s'accompagnant au piano et étant soutenu par une partie de l'orchestre. La troisième est entièrement occupée par un sermon de Jimmy Swaggart.

Les trois parties de cet office durent respectivement trente-cinq minutes, six minutes et demie et une heure, dix-neuf minutes ${ }^{9}$.

\section{Première partie : Jazz et ambiance}

L'émission ouvre sur une image présentant en plan large une vue d'ensemble sur l'intérieur de la salle. Cette vue montre une salle entièrement occupée par des hommes et des femmes. En surimpression, le visage du chanteur John Starnes, un homme d'une quarantaine d'année dont la large calvitie est entourée de cheveux blonds. Il chante On Jordan Stormy Banks (I am Bound for the Promised Land ${ }^{10}$ )dont le début est un blues lent et doux qui deviendra rapide et syncopé. L'artiste est accompagné par l'orchestre et le Family Worship Center Choir ${ }^{11}$. Au moment du changement de rythme, un autre chanteur se joindra au premier : Dudley Smith, d'une quarantaine d'années lui aussi, est noir. La salle se lèvera peu après l'accélération du rythme et les spectateurs soutiendront le chant et l'orchestre, soulignant la cadence en frappant dans les mains. L'ambiance s'échauffera au fur et à mesure que se déroulera le chant et l'on verra des gens se déplacer, bouger leur corps au son de la musique et agiter les bras. 
L'interprétation de ce chant dure sept minutes et quinze secondes. À ce moment, Jimmy Swaggart entre sur la scène. Il tient un microphone par le pied. Nous voulons dire que le microphone n'est pas désolidarisé de son support comme cela pourrait se concevoir. Jimmy Swaggart est le seul à tenir ainsi son micro, les chanteurs n'utilisant pas de pied et les choristes du petit chœur ne touchant pas aux leurs : on verra que cette façon de tenir le micro s'apparente à un comportement codifié et, comme tel, connu des spectateurs et téléspectateurs. Il crie " Alléluia $^{12}$ » et invite l'orchestre et les chanteurs à reprendre. Ce qu'ils font. Il recommencera une seconde fois lorsque les chanteurs s'arrêteront, après s'être écrié : «Oh, Mamma! That's the best cure for nervous break down in the world ${ }^{13}$ !» Il recommencera une troisième fois. Ces relances constituent une véritable excitation, un encouragement, quelque chose qui se rapprocherait de ce que les publicitaires appellent le « teasing » et qui consiste à agacer le public-cible par une répétition du stimulus.

Lorsque la chanson prend définitivement fin, Jimmy Swaggart entre à nouveau sur la partie avancée de l'espace scénique. Il traverse la piste en tenant le microphone d'une main, par le pied, geste habituel des chanteurs de rocks, et tout comme le font ceux-ci, en apostrophant la foule qui répond:

«Pourquoi ne dîtes-vous pas Alleluia?

- Alleluia!

- Pourquoi ne dîtes-vous pas « Jésus, je vous aime»?

- Jésus, je vous aime!

- Pourquoi ne dîtes vous pas : « Avec le seigneur, je suis libre »?

(Applaudissements de la salle)

- Vous pouvez vous asseoir. «

Jimmy Swaggart lui-même annonce: «Ladies and Gentlemen, Dudley Smith». Le chanteur noir que nous venons de voir entre en scène à nouveau. Il interprète un air très rythmé et qui rappelle certains negro spirituals traditionnels très chauds que les amateurs de jazz connaissent bien (on pense au célèbre When the saints,par exemple). Cette chanson, qu'il a écrite avec Jimmy Swaggart et un troisième auteur du nom de Roger Price, s'intitule That's the kind of God that I serve(Voici le genre de Dieu que je sers).

37 L'un des invités, présenté sur la jacquette de la cassette comme R.W. Schambach, particulièrement stimulé par la musique ainsi que trois plans nous l'ont montré, finira par rejoindre Dudley Smith pour un duo particulièrement dynamique. Il est vêtu d'un pantalon bleu clair, d'une veste jaune et d'une cravate claire sur une chemise blanche. Les deux chanteurs se font face et se répondent comme en une surenchère, se pointant réciproquement du doigt en affirmant une des particularités du Dieu qu'il servent. On pourrait presque qualifier ce duo d'agressif si les visages des interprètes n'étaient aussi ouverts et souriants.

38 Le public est très énervé, tous les fidèles frappent dans les mains, la plupart chantent, certains courent et sautent, bras levés. Certains choristes ont quitté leur place et courent dans la salle.

À trois reprises, Jimmy Swaggart invitera les chanteurs à reprendre et il le fera en des termes qui ne sont pas de nature à calmer la foule : « Once more! Once more ! Once more, men!» (Encore une fois! encore une fois! encore une fois !). Si bien que ce chant durera vingt et une minutes et trente secondes! A la fin de ce duo, semble-t-il improvisé, Smith 
et Schambach tomberont dans les bras l'un de l'autre et se congratuleront avec de grands rires à la manière des jazzmen heureux d'avoir pu « faire un bœuf ${ }^{14}$ » ensemble.

On est frappé par l'usage fréquent que les acteurs font de la répétition incantatoire. A ce « once more ! » trois fois répété répond, par exemple, le triple « Jesus is here ! de Dudley Smith.

41 On ne peut pas non plus ne pas remarquer le rôle d'animateur que Jimmy Swaggart se réserve. Il présente les chanteurs, il les relance, il commente leurs performances. Il entretient l'enthousiasme du public par des formules non moins enthousiastes et qu'il crie plus qu'il ne les dit.

\section{Deuxième partie : Sous le charme du crooner}

Après avoir invité la foule à s'asseoir, Jimmy Swaggart s'installe au piano. Il joue et parle sur le fond sonore ainsi créé pour présenter It's beginning to rain,une chanson dont il est l'auteur en collaboration avec trois autres personnes. Il dit dans quelles circonstances lui sont venues les Paroles. Puis les percussions et les claviers jouent l'introduction.

Jimmy Swaggart, cadré tantôt en gros plan et tantôt en plan américain, chante d'une voix qui s'apparente à celle des chanteurs de charme, les fameux « crooners » américains. On pourrait rapprocher son interprétation aussi bien que le timbre de sa voix de ceux du célèbre chanteur Franck Sinatra. Le rythme est lent et balancé. Il correspond, dans la nomenclature des rythmes inscrits sur les partitions, à un slow.

Cette chanson mélodieuse et douce a pour effet de calmer le public, de l'apaiser après l'excitation qu'il vient de connaître pendant trente-cinq minutes et à laquelle Jimmy Swaggart lui-même avait pris part. On peut dire qu'elle sert de transition et permettra d'écouter le sermon dans le recueillement.

Il n'y a pas la moindre irrévérence dans le rapprochement entre Jimmy Swaggart et Franck Sinatra. Tout au contraire, une oreille un peu exercée reconnait chez le pasteur de Baton Rouge de réelles qualités de chanteur et un grand professionnalisme. Ce que confirme sa discographie: Jimmy Swaggart est son propre éditeur en tant que propriétaire de sa maison de disques et de cassettes sonores, JIM RECORDS, ainsi que de sa maison de production de vidéo, JIM VIDEO ALBUMS.

La qualité de l'interprétation vocale de It's beginning to rainaussi bien que son orchestration et son accompagnement musical et vocal nous permettent effectivement de conclure que l'on est en présence d'un artiste professionnel dont le talent est amplifié par une technique avérée. Nous ne voudrions pas oublier, à ce propos, la qualité de la technique en salle: qu'il s'agisse du son ou de l'image, la cassette vidéo que diffuse la société JIM VIDEO ALBUMS donne toute satisfaction à l'acheteur.

\section{Troisième partie : Le sermon}

47 S'approchant du pupitre placé sur la piste, Jimmy Swaggart invite ceux qui ont une bible à l'ouvrir au Livre des Premiers Rois,chapitre XVII, Versets 1 à 3. À noter que cette indication apparait en incrustation dans la version télévisée de l'office. Il annonce le titre de son sermon, Cherith, Carmel and the Cave, lequel titre apparaît lui aussi en incrustation à l'écran, et avertit ses auditeurs: " Somewhere in here, you are there. Somewhere in the scenario, you will find yourself ${ }^{15}$ » Puis il implore l'aide de Dieu : « Père, aide moi ce soir. » 

à lui seul, il dure deux fois plus longtemps que l'ensemble des deux premières parties. Qualitativement, il constitue la raison du rassemblement des fidèles. Dans ces religions d'inspiration protestante qui prônent la communion - communication - directe avec Dieu, dans cet office où, contrairement à la messe des catholiques, on ne célèbre pas de mystère, c'est la lecture de la Bible, son interprétation et son extrapolation qui constituent la raison d'être du rassemblement des fidèles. On comprend dès lors que la fréquentation du temple soit en relation directe avec le talent du prédicateur. N'oublions pas que, dans les années 50 en France, la prédication du carême à Notre-Dame de Paris était annoncée par affiches et que fidèles et autres se pressaient pour écouter tel prédicateur plutôt que tel autre. Le Révérend Père Riquet était, si l'on ose dire, une vedette qui attirait les foules.

L'analyse des textes de quelques sermons de Jimmy Swaggart, que nous avons pu faire à partir des documents officiels ${ }^{16}$ servant à la post-synchronisation française de ses cassettes, montre le peu de contenu sémantique des dits sermons. En fait, le talent du télévangéliste réside plus dans l'interprétation que dans l'argumentation. Il apostrophe son public, il le met en accusation, il l'interpelle et cela le dispense d'une logique argumentative. Nous sommes dans une rhétorique de séduction où compte plus la bonne impression laissée que la qualité de la démonstration. À titre indicatif, nous donnons ciaprès un extrait d'un sermon intitulé L'aveugléné, prononcé en décembre 1992. On pourra ainsi avoir une idée du contenu anecdotique et de la forme fondée sur la fonction conative du langage ausi bien que sur l'intention phatique ${ }^{17}$ de la communication.

Gloire ! v Vous recevez plutôt mal mon message, ce soir./ Vous recevez mal mon message, $\mathrm{V}^{18} \mathrm{Et}$ pour ceux qui nous suivent à la télévision, regardez, $\mathrm{v}$ peu importe qui vous êtes. Vous pouvez avoir pris une forte dose de drogue $\mathrm{v}$ au point que votre esprit même est en difficulté, il ne reste rien dans cette chambre, il n'y a plus de meubles, v vous êtes assis sur un vieux matelas, v l'enfer hurle vers vous, vous voyez des démons $v$ et vous avez tant bu que vous voyez des éléphants roses $v$ et des tigres rouges et des animaux hurlant après vous et Satan dit : «Il n'y a plus d'espoir.» v Mais si vous laissez Jésus v venir à vous, permettez cela, v laissez Jésus s'occuper de vous, v II repoussera les démons, v fera reculer l'enfer, v parce qu'il est venu pour cela.v II est venu pour cela ! v Venu pour cela! $\mathrm{v}$ (applaudissements) Il est venu défaire ce que le diable a fait, $\mathrm{v}$ Affranchir les captifs. Apporter la lumière, chasser les ténèbres, v II est venu vous libérer ! (réponse du public) (applaudissements)

51 Je vais déranger les gens qui n'ont que la religion./ Si vous avez eu huit maris, madame, il vous sauvera./ (réponse du public) Je sais qu'il le fera./ Une femme/ en avait eu cinq./ (réponse du public) Et elle cessait de compter, (rires) C'est sérieux ${ }^{19}$./ Elle a dit : «Je ne crois pas que je sois mariée à celui avec qui je vis. »/(réponse du public) Et je vais, v je vais malmener les principes que vous prônez et qui vous donnent bonne conscience./ Vous ne voyez pas très bien de quoi je parle, n'est-ce pas ?/ Je vais mettre à mal vos principes./ La pauvre petite femme qui avait été mariée cinq fois $\mathrm{v}$ est devenue la première femme évangéliste. $v$ (réponse du public) Gloire à Dieu ! (applaudissements) Elle courut en ville, $\mathrm{v}$ disant : «Je veux vous parler d'un homme! $\mathrm{v}$ Je veux vous parler d'un homme ! v Je veux vous parler d'un homme v qui a sauvé mon âme ! v II a changé ma vie! v II m'a élevé au-dessus des ombres ! v II m’a élevée ! Il m'a dit tout ce que j'étais ! v II a mis la paix dans mon cœur! v Je veux vous parler d'un homme!/ (applaudissements) (réponse du public) (applaudissements) Jésus ne peut faire beaucoup aux petits 
religionnistes pieux ${ }^{20}$./ (rires) Vous allez à l'église tous les dimanches matin./ (rires) (applaudissements) Vous vous y asseyez!/ (rires) (applaudissements) Oh, vous êtes si religieux!/ (rires) Ne relevez pas trop la tête, il pleuvrait dans votre nez/ (rires) (applaudissements) Entendez-vous ce que je dis. Je sais $v$ que je suis en train de troubler certains d'entre vous. Je réalise que certains vont dire $\mathrm{v}$ « Au fond, $\mathrm{v}$ il n'aurait pas dû dire cela! «Je sais, v parce que vous êtes l'un d'eux!/ (rires) (applaudissements)(réponse du public) Qui est Jésus selon votre opinion? v Qui est-Il ? Pensez-vous qu'il chante dans la chorale ? v Pensez-vous qu'il se tient derrière un podium avec des vêtements spéciaux? v Selon vous, serait-il comme cela? Peut-être bien, v mais je vais vous dire aussi ce qu'il est et où il est! v II annonce la bonne nouvelle aux pauvres ${ }^{21}$ ! v II soigne, il guérit les cœurs brisés ! v II rend la liberté à ceux qui sont opprimés ! v II libère les prisonniers ! v II s'appelle Jésus !/ (applaudissements) (réponse du public)

Les dominantes de cette interprétation sont :

- la pontuation du discours par les éclats de rire et les «Alleluia» du prédicateur. À chaque fois, les fidèles répondent en levant la main. Le visage de Jimmy Swaggart, rond et plein avec des yeux enfoncés, n'est pas très expressif et seul le rire le marque vraiment. Des expressions telles que la gravité, l'enthousiasme, la colère, n'y trouvent pas place et les émotions du télévangéliste transparaissent peu, même en plan rapproché.

54 - la récurrence de deux mouvements qui dominent une gestuelle foisonnante: le doigt levé ou le doigt pointé vers une personne en particulier ou une partie du public. Cette attitude s'accompagne souvent d'une augmentation du volume sonore de la voix.

- la récurrence de deux attitudes : debout, bible ouverte dans la main droite levée

- et l'on notera qu'il reproduit ainsi le logo de son entreprise Jimmy Swaggart Ministries inc - ou bien traversant la scène à grands pas en tenant le microphone par le pied.

- les facéties qui émaillent le prêche. Il feint de compter les mots de la Bible qu'il tient en mains et éclate de rire, il prend des poses comiques en appui sur son pupitre et, là aussi, éclate de rire. Ou bien encore, il prend le public à témoin et, levant les yeux au ciel : «Je ne sais pas si vous êtes mariés... moi, je le suis !» (la caméra nous gratifie alors d'un gros plan sur le visage souriant de son épouse Frances).

58 Tout le sermon se déroule sur ce mode à la fois familier et pseudo-pédagogique. Le statut que le prédicateur se donne est celui du maître qui explique à ses disciples. Un peu comme Jésus, dans Les Évangiles, expliquait la parole du Père en usant de paraboles, Jimmy Swaggart semble expliquer la Bible à ses propres disciples. Dans les faits, il harangue plus qu'il ne donne à comprendre, il s'exclame plus qu'il ne dit, il reproduit plus qu'il n'improvise ses attitudes. Pour ce dernier point, il évoque ces comédiens dont le jeu simplifié à l'extrême facilite la réception et autorise une reconnaissance immédiate d'un lexique visuel et sonore réduit. On pense à Louis De Funès bien que Jimmy Swaggart ne lui ressemble nullement. Mais tous deux développent une gamme réduite de stimuli éprouvés faits pour une lecture rapide des émotions interprétées. Ainsi procèdent aussi les catcheurs, les artistes de cirque et les acteurs de feuilletons télévisés américains dont on peut dire que... quand ils pensent, cela se voit. En ce sens, Jimmy Swaggart développe une démarche d'acteur qui s'inscrit dans les traditions du spectacle populaire, ce qu'ailleurs nous avons appelé "spectacle à lecture première ${ }^{22}$ ", fondé sur la stimulation d'un registre limité d'émotions simples que le spectateur mobilise dès lors qu'il prend place dans le fauteuil de son salon, dans l'amphithéâtre du cirque, dans l'arêne du catch ou dans l'église-sallede spectacle du Family Worship Center. Car, bien entendu, le 
spectateur n'est ni innocent ni passif et, connaissant bien le canevas de l'office-spectacle, le contenant, il ne lui reste qu'à bénéficier de la surprise du contenu selon la formule de Robert Escarpit qui définit le plaisir de la découverte informationnelle par l'équation : minutes de rires, de cris d'assentiments, d'applaudissements aux bons mots de l'orateur, les fidèles sont invités à se recueillir. Jimmy Swaggart les prie de se lever et appelle sur eux les bienfaits de Dieu, bienfaits qu'il détaille tandis que l'orchestre joue en sourdine. Le prédicateur alternera les parties chantées et les parties parlées. Il chante avec le public et le chœur mais, effet de sonorisation, sa voix domine comme celle d'un soliste qui couvrirait l'orchestre. Jimmy Swaggart s'adresse directement au public: "Je sais que certains parmi vous ont besoin de Lui. Ils ont faim, ils ont soif, ils sont découragés. » La caméra nous montre longuement le visage d'un jeune homme borgne, portant un bandeau sur l'oœil gauche et fermant l'autre dans une attitude de recueillement. Des fidèles se lèvent, s'avancent vers la piste octogonale, s'agenouillent et se prosternent. D'autres, debout derrière eux, baissent la tête. Jimmy Swaggart marche, chante et, à deux reprises, éclate de rire. Tous les spectateurs, même ceux qui sont restés à leur place, ferment les yeux et se recueillent. Ainsi se terminent l'office et l'émission. et étrangères et vendue en version VHS dans la boutique de l'église a inséré des gros plans de visages de fidèles recueillis et/ou émus. Nous avons dénombré huit plans de couples dont un d'un adulte et d'une jeune fille (père et fille?) et un d'une adulte et d'un jeune homme (mère et fils?), onze plans de visages masculins et dix-sept plans de visages féminins. La majorité des femmes présentées sont relativement jeunes, entre trente et quarante ans semble-t-il, coiffées et maquillées avec soin ${ }^{24}$. Il émane de leur visage une émotion que la beauté et le maquillage amplifient.

63 L'office est conçu comme un spectacle de music-hall, avec une progression qui contribue à glorifier la star. Simple animateur de concert de jazz au début, il s'impose ensuite comme artiste dans un autre genre, le charme, avant d'être lui-même, celui qu'on attend, le prédicateur vedette de la télévision. Toute la communication de la star repose sur son charisme car tout est fait pour mettre sa personne en valeur. Cela est si vrai que lorsqu'il fut suspendu pour deux ans par Les Assemblées de Dieu et que son fils et d'autres prédicateurs le remplacèrent, le public bouda au point de compromettre les finances de l'entreprise Jimmy Swaggart Ministries inc. Il faut bien parler, en l'occurrence, de spectateurs plutôt que de fidèles car ce comportement s'apparente plus à celui d'un consommateur de spectacle «fan » d'une star qu'à l'attachement religieux d'un fidèle à une foi.

Beaucoup de religions ont tenté d'induire les fidèles par une spectacularisation de la liturgie. L'Église catholique romaine a usé et abusé des couleurs et des formes, de la parole et des chants, quand ce n'était pas des odeurs de l'encens, pour induire, conditionner et assujettir les participants aux offices. La liturgie romaine s'est dépouillée depuis le concile Vatican II mais il est d'autres religions chrétiennes qui n'ont guère changé. C'est, par exemple, le cas chez les orthodoxes. La différence capitale est que les grandes religions, quand elles recourent à une liturgie spectaculaire, le font pour subjuguer les fidèles afin de les rendre plus disponibles pour Dieu. À l'évidence, le 
télévangéliste qui utilise la puissance de son charisme pour prendre un ascendant sur les fidèles le fait à son propre bénéfice. Et force est de constater que le mot vaut ici dans toutes ses acceptions tant l'argent est omniprésent chez ces nouveaux prédicateurs.

\section{«Church marketing »}

65 Le rapprochement de ces deux mots choquerait sans doute le lecteur s'il nous était imputable. C'est, en fait, le titre d'un ouvrage ${ }^{25}$ que l'on peut se procurer dans les librairies spécialisées en littérature religieuse. Nous l'avons acheté au Gospel Book Center de Gretna, Louisiane. La carte de ce magasin désigne John \& Essie Smith comme « stewards " et Jésus Christ comme propriétaire (« owner »).

Les Français voient toujours d'un mauvais œil les rapports entre la religion et l'argent. Peut-être parce que le Christ était pauvre parmi les pauvres et chassait les marchands du temple, ils sont choqués par le fait que la religion puisse être conçue comme une source de revenus. Surtout si'il s'agit de revenus élevés. Les Américains n'ont pas cette pudeur. Dès lors qu'on est honnête, on peut faire feu de tout bois et argent de toute industrie. Pour avoir évoqué l'argent gagné par le célèbre Billy Graham avec ses prédications, l'auteur de cette étude s'entendit répondre qu'il n'y avait pas de honte à gagner beaucoup d'argent en prêchant la gloire de Dieu pour peu qu'on le fit honnêtement. Sans que cela gêne le moins du monde l'esprit américain, les églises sont, répétons le, des entreprises qui se gèrent et se transmettent comme n'importe quelle autre affaire. Jimmy Swaggart a un fils pasteur présent à ses côtés et qui est un successeur potentiel. Dans sa livraison du 13 mai 1996, le sérieux Time présente en couverture Billy Graham les mains posées sur les épaules de son fils avec pour titre "The prodigal son ». Le sommaire du magazine résume ainsi l'article de huit pages qu'il consacre au retour de l'enfant prodigue et qu'il intitule Au nom du Père :

67 «Franklin Graham était né pour devenir né à nouveau et pour hériter du Royaume évangélique de son père Billy. C'était un destin tout tracé pour l'ainé des fils Graham, mais il se rebella contre ce fait. C'est ici l'histoire de sa rédemption et comment il se tient prêt à reprendre l'affaire familiale ${ }^{26} »$.

68 Cette mise au point est importante car il convient toujours d'analyser une production, culturelle ou communicationnelle notamment, dans son propre contexte et non dans celui de l'analyste. Là où nous voyons cynisme et manque de retenue, les Américains ne voient rien d'autre qu'un « business » comme les autres. Et il ne faudrait pas imputer à la religion des comportements qui, en fait, sont culturels.

69 C'est donc avec prudence que nous livrons quelques uns des éléments qui figurent sur la couverture du livre dont il est ici question. Nous les présentons sans commentaire sauf à dire que c'est sans hypocrisie que la religion est considérée comme faisant partie du domaine des affaires :

\section{Couverture}

Comment établir des stratégies de marketing efficaces fondées sur la Bible et menée d'une façon chrétienne 
Comment diriger la partie affaire [the business side] de votre église sans compromettre votre intégrité spirituelle

\section{Dernière de couverture}

Bien que le mot " marketing " n'y apparaisse jamais, la Bible est pleine d'exemples de spécialistes du marketing [marketers] qui ont connu le succès: Salomon, Joshua, Nehemiah et Paul, pour n'en citer que quelques uns. Comme vous le lirez dans ce livre, ces hommes de Dieu dynamiques avaient la capacité d'identifier les besoins des gens et de trouver les meilleurs moyens d'aller à leurs rencontres.

\section{(...)}

Il est temps de vous départir [to move beyond] de la question de savoir si le marketing est ou n'est pas une pratique biblique; la véritable question est de vous demander si votre église peut se passer d'un outil si puissant et si accessible.

La communication de l'entreprise religieuse avec ses membres est principalement axée sur la demande de dons. Après la diffusion télévisée d'un office, le prédicateur revient à l'écran et lance un appel, à moins qu'un spot publicitaire ne propose des articles produits par le prédicateur: livres, cassettes, disques, vidéo. Les procédés diffèrent de l'un à l'autre. Jimmy Swaggart expose les factures de son église et demande qu'on l'aide à les payer. Le président de la Liberty University offre d'envoyer gratuitement deux "pins » (épinglettes) à qui lui communiquera le nom et l'adresse d'un garçon ou d'une fille présentement en collège (high school = cycle préparatoire aux études universitaires).

Les journaux que diffusent les télévangélistes aux adeptes sont vendus par abonnements. Ils comportent des articles généralement centrés sur l'activité du prédicateur. Parfois, ce sont des questions posées par des lecteurs et les réponses fournies par le chef de l'église. La publicité y tient une large place. The Evangelist,l'organe de l'église de Jimmy Swaggart, est un journal de 20 pages en format voisin du A4 européen. Sept pages sont consacrées à la publicité. Hormis une colonne pour une agence de voyage, tous les produits et services proposés émanent de la Jimmy Swaggart Ministries (association à but non lucratif la non profit organization]). Ces journaux proposent des produits mais lancent aussi des appels de fonds souvent pathétiques. Dans sa livraison de novembre 1992, The Evangelistsous le titre Courrier volé !informe ses lecteurs que nombre de leurs chèques ont été détruits ou volés pendant le transfert postal. Après un vibrant appel à l'aide: «Dans les derniers mois, nous avons frôlé la faillite et la situation continue d'être dangereuse. La situation a été critique ! Elle l'est toujours! " [The situation has been critical! The situation is still critical !] Et l'inspirateur de ces vols est dénoncé nommément. Après avoir rappelé l'œuvre accomplie, le texte prend des majuscules pour affirmer : «C'EST POUR TOUTES CES RAISONS QUE SATAN NOUS COMBAT SI RUDEMENT ! » 


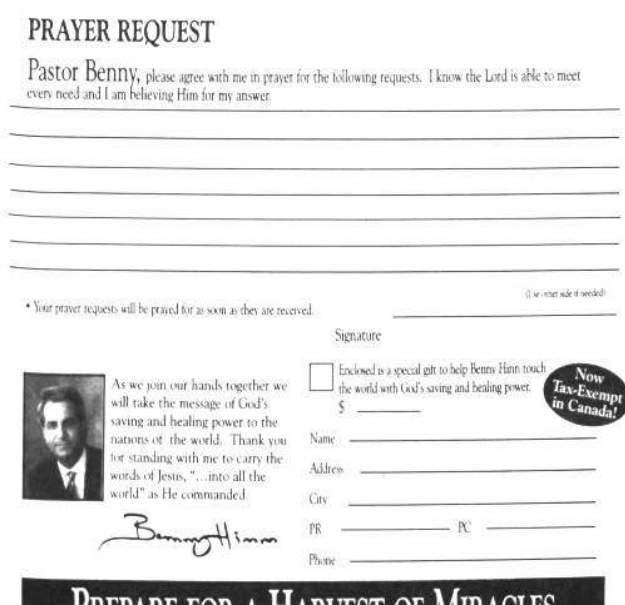

\section{Prepare FOR A HaRVEST OF MiraCles}

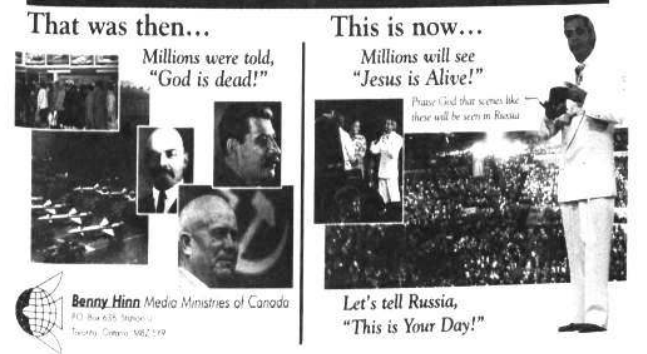

78 Un autre moyen de contact avec les prospects, « born again » ou supposés sympathisants, est l'envoi en nombre de circulaires plus communément appelé mailing. Les fichiers sont obtenus à partir des commandes, des demandes de renseignements et des adresses collectées à l'issue des offices. L'un des expéditeurs de circulaires est Benny Hinn, un prédicateur installé à Orlando, Floride, qui est connu pour sa "Croisade des miracles ».Il parcourt les États-Unis et, dans des salles immenses, généralement dédiées au hockey, il réunit des foules de dix à quinze mille personnes qui viennent le voir accomplir des miracles en direct. Chacune de ses lettres comporte une très bonne nouvelle. Par exemple - circulaire de mai 1995 - qu'il va enfin pouvoir évangéliser la Russie. Rien n'est caché au lecteur. Pas même le prix de la production et de la diffusion des programmes : 250000 \$ par an. Enfin, un appel est lancé pour que les fidèles contribuent largement à cette mission. En contrepartie, il est vrai, ils pourront indiquer au Pasteur Benny un motif particulier, et personnel, de prière. Et il priera le Seigneur pour que la demande soit exaucée.

Certains vont encore un peu plus loin et n'hésitent pas à recourir à la culpabilisation et au chantage. Si le «born again » n'a pas répondu à la quête, il sera avisé que, par sa faute, des âmes ne seront pas sauvées et qu'il aura à en rendre compte directement à Dieu...

Ilfaudrait connaître la Bible mieux que nous ne la connaissons pour dire si le marketing est une pratique biblique. Mais il est clair qu'à la question de George Brana, la réponse est aisée : oui, manifestement, les églises dont il est question ont besoin de recourir au marketing et celui-ci semble suffisamment efficace pour assurer la rentabilité de l'entreprise.

81 Nous supposons, bien entendu, que les prédicateurs dont nous parlons sont d'authentiques zélateurs du Dieu de la Bible. C'est donc à son service qu'ils mettent leurs compétences et leurs efforts. Le charisme du télévangéliste est une de ses particularités les plus évidentes. En même temps qu'il œuvre à la gloire de Dieu, il assure sa propre 
renommée. Celle-ci fait prospérer l'entreprise qu'il a créée au service de Dieu et lui permet généralement un train de vie qui, dans une société de la possession et des signes extérieurs de richesse, ne peut que contribuer à la gloire du Seigneur. La communication est le fondement même de ces organisations. C'est elle qui assure tout à la fois la transmission du message divin, la notoriété du messager et les moyens de poursuivre l'œuvre. Nombre de chefs d'entreprise seraient avisés de méditer ce fonctionnement d'une rare efficacité et qui donne satisfaction à tous sans exception. Grâce à Dieu.

\section{BIBLIOGRAPHIE}

BALADIER Charles Le grand atlas des religions, Paris, Encyclopedia Universalis, 1990

BARNA George A step by step guide to Church Marketing, Ventura, Ca, USA, Regal Books, a division of Gospel Lights, 1992

BERTRAND Claude-Jean Les églises aux États-Unis, Paris, PUF, collection Que sais-je ?, 1975

BOOTH Leo (Father) When God becomes a drug, Los Angeles, Ca, USA, Booth, 1991

BRYANT Al. Sermon outlines for worship services, Grand Rapids, Mi, USA, Kregel Publications, 1992

CARLANDER Ingrid Les stars de Dieu, Paris, Plon, 1990

CLARKE Peter B. Le grand livre des religions du monde, Paris, Solar, 1995

COLSON Charles W Born Again, Grand Rapids, Fleming H. Revel, vingt-et-unième édition, 1993

ENROTH Ronald M. Churches that abuse, Grand Rapids, Mi, USA, Zondervan Publishing House, 1992

GRAHAM Billy Angels God's secret agents, Billy Graham, 1975

GURWITH Jacques Les Judéo-chrétiens aujourd'hui, Paris, Cerf, 1987

HARRELL Jr David Oral Roverts, San Francisco, Harper and Row, 1985

HOUSE Wayne H Christian ministries and the Law, Grand Rapids, Baker Book House, 1992

KANTROWITZ BarbaraCette vague qui soulève l'Amérique in L'actualité religieuse, Paris, mars 1996

LEAVITT Mel A short history of New Orleans, San Francisco, Lexicos, 1982

POUPARD Paul Les religions, Paris, PUF, collection Que sais-je ?, 1987

SCHMIDT Rosemarie et KESS Joseph FTelevision advertising and televangelism, Amsterdam/

Philadelphia, John Benjamins Publishing Company, 1986

STRAUB Gérard T Salvation for sale, New York, Promotheus Books, 1988

VALADIER Paul L'Église en procès, Paris, Flammarion, 1989

\section{NOTES}

1. Il est vrai que, bien que Démocrate, Jimmy Carter était « born again » lui aussi 
2. Les stars de Dieu Paris, Plon, 1990

3. Gris Gris, 18 septembre 1985

4. Ibid.

5. Les stars de Dieu, op.cit., page 56.

6. Time, 11 septembre 1986

7. Time, 28 octobre 1991. « The Lord told me : «It's flat none of your business »

8. Cirque, communication, culture, Presses Universitaires de Bordeaux, 1995.

9. Nous avons systématiquement arrondi à trente secondes ou à la minute entière les durées exprimées.

10. Il s'agit d'un negro spiritual traditionnel. La jacquette de la cassette vidéo que nous utilisons précise que l'arrangement est de Jimmy Swaggart.

11. Dénomination du chœur telle qu'elle apparaît sur la jacquette de la cassette vidéo.

12. Vient de l'hébreu et signifie littéralement "Louez le Seigneur ", cri de joie des religions chrétiennes.

13. "Oh, Maman ! c'est ce qui se fait de mieux au monde contre la dépression nerveuse !»

14. Expression que les musiciens de jazz emploient pour désigner les interprétations improvisées qu'ils font, pour leur propre plaisir, quand ils se rencontrent.

15. "Quelque part ici, il y a vous. Quelque part dans cette histoire, c'est vous que vous trouverez.»

16. En 1992, lors d'un séjour à Baton Rouge, il nous a été possible de nous procurer les traductions officielles de plusieurs sermons. Jimmy Swaggart était doublé, dans la version française de ses émissions, par André Josset, pasteur d'une église évangélique en France et qui séjournait régulièrement en Louisiane pour enregistrer les post-synchronisations.

17. Avec Greimas et Courtes, nous parlerons ici d'intention ou d'acte phatique plutôt que de fonction phatique. Voir notamment Greimas A. J. et Courtès J. Dictionnaire raisonné de la théorie du langage, Paris, Hachette, 1979.

18. Les marques portées dans le texte (soulignage, $\mathrm{v}, /$ ) ainsi que les indications portées entre parenthèses sont dans le document remis au pasteur Josset et (ont partie des indications dont il a à tenir compte En fait, ce texte n'est pas préécrit, il est une transcription du sermon filmé en public par la société Jim Video Albums.

19. I'm serious

20. Jesus can't do much with you little pious religionists Le traducteur propose une variante : Jesus ne peut faire beaucoup pour ceux qui sont très religieux.

21. He is preaching the gospel to the poor!

22. Signes du cirque, approche sémiologique Association Internationale de Sémiologie du Spectacle, Bruxelles, 1984

23. Théorie générale de l'information et de la communication. Hachette, Paris, 1976

24. D’après une étude Gallup réalisée en 1985 et portant sur les dix télévangélistes les plus suivis, l'audience de Jimmy swaggart est majoritairement composée de femmes du Sud, de race blanche, âgée de 25 à 54 ans.

25. George Barna A step-by-step Guide to Church Marketing Breaking Ground for the Harvest Ventura, California, USA, Regal Books, a division of Gospel Light, 1992

26. Franklin Graham Was born lo be born again and to inheri the Evangelical kingdom ot his father Billy. It was,in overwhelming fate lor the Graham's first-born son, and the rebelled against it. This is the story ot his redemp tion and how he poised to take over the fammilv business. 


\section{RÉSUMÉS}

Héritiers des prédicateurs itinérants des XVIIIe et XIXe siècles, les télévangélistes touchent leurs ouailles par le biais de la télévision. Leur communication est fondée sur une maîtrise parfaite de ce média qui convient bien à la parole publique. Les églises des télévangélistes sont généralement des entreprises importantes qui ont besoin d'argent. Les techniques du marketing sont donc fréquemment utilisées pour les levées de fonds. Communication charismatique et marketing sont les deux aspects d'une communication qui est ici étudiée.

Heirs of the ancient preachers who travelled up and down during the XVIII ${ }^{\text {th }}$ and XIX ${ }^{\text {th }}$ centuries, the televangelists reach their flock by the meaning of television. Their communication is based on a perfect knowledge of this good for public speech medium. The churches of the televangelists are big enterprises and need a large amount of money and they use very often the techniques of marketing for their funds raising-Spectacular production and marketing are two aspects of a profitable communication.

\section{AUTEUR}

\section{HUGUES HOTIER}

Professeur à l'Université de Bordeaux 3 où il enseigne les stratégies de communication et les méthodes en audit à l'Université de Bordeaux 3 où il dirige l'Institut des Sciences de l'Information et de la Communication et le Groupe de Recherche en Communication des Organisations. Ses recherches actuelles portent sur les processus d'induction, notamment dans la communication charismatique des télévangélistes. 